\title{
A NEW METHOD FOR ASSESSING HAEMOLYSIS IN A ROTARY BLOOD PUMP USING LARGE EDDY SIMULATIONS (LES)
}

\author{
Maciej Szwast ${ }^{*}$, Arkadiusz Moskal, Wojciech Piątkiewicz \\ Warsaw University of Technology, Faculty of Chemical and Process Engineering, Waryńskiego 1, \\ 00-645 Warsaw, Poland
}

\section{Dedicated to Prof. Leon Gradon on the occasion of his 70th birthday}

\begin{abstract}
The opportunity to assess haemolysis in a designed artificial heart seems to be one of the most important stages in construction. We propose a new method for assessing haemolysis level in a rotary blood pump. This method is based on CFD calculations using large eddy simulations (LES). This paper presents an approach to haemolysis estimation and shows examples of numerical simulation. Our method does not determine the value of haemolysis but allows for comparison of haemolysis levels between different artificial heart constructions.
\end{abstract}

Keywords: rotary blood pump, large eddy simulations, haemolysis

\section{INTRODUCTION}

Over the decades pulsatile pumps have been used to assist a diseased heart. Their pulsatile operation resembles the work of the natural heart. The most popular pulsatile pump in the world has been a membrane pump with pneumatic drive, which was used for the first time in 1982 by Jarvik (Leprince et al., 2003). In clinical practice, it has been used to this day.

Currently, one can note a growing interest in centrifugal pumps. Such pumps provide a continuous flow of blood. It is noted that the pulsating flow, despite its physiological nature, is not so important when using artificial ventricles (Garatti et al., 2008; Saito and Nishinaka, 2005). The advantage of centrifugal pumps is their small size, which in the future may lead to their total implantation. Another advantage of these pumps is the lack of the diaphragm and valves, failure of which may lead to a sudden death of the patient.

There are two main types of rotary pumps: axial and centrifugal. In the first type of pumps, blood flows along the axis of the pump. An impeller rotating around the axis of the pump causes the flow. In the second type, i.e. centrifugal pumps, the impeller blades move blood, and thus the blood ejection is caused by the centrifugal force. The efficiency of rotary pumps depends on the rotor speed, which for this type of pumps, is several thousand revolutions per minute.

There are many aspects that must be considered while designing an artificial heart. The constructor should have in mind problems such as reliability, material haemocompatibility, chamber's size, heat exchange and many others. But one of the most important issues is whether the artificial heart will not damage blood cells. It is known that in rotary blood pumps high mechanical stress may occur inside the chamber. 
In the artificial heart ventricle a centrifugal pump with the rotating impeller creates areas of high values of hydrodynamic stress. This physical stress may affect blood cells and can result in blood cell deformation and damage. The literature presents many hypotheses of the destruction of Red Blood Cells (erythrocytes) in the blood flow. The literature also gives different, often very divergent, values of stress that cause cell damage.

A Red Blood Cell (erythrocyte) is a specific cell. It does not contain a cell nucleus. Its task is to transfer oxygen between the alveoli and all the cells of the body. Gas transport is possible thanks to the protein haemoglobin which is dissolved in the cytoplasm of red blood. The destruction or leakage of the erythrocyte membrane causes the release of haemoglobin into the circulating blood plasma. High concentrations of free haemoglobin and in particular of its degradation products, such as bilirubin, are strong poisons for the body. Therefore, there is a need to prevent the destruction of red blood cells, i.e. haemolysis during the application of artificial organs and in particular of blood pumps.

Inside a centrifugal pump, one can expect mostly physical stresses affecting the red blood cells. These stresses are caused by hemodynamic effects, such as the collision of cells with the impeller blades or with the pump housing. Such stresses can also be a result of a mutual cell collision or because of cell flow interactions (Pohorecki et al., 2001).

As a result of this stress, the cells may be destroyed. Threshold values for such a stress that erythrocytes can resist, are not clearly defined. The literature gives a number of figures for these stresses - from $10^{2}$ to up to $10^{4} \mathrm{~Pa}$. These values were determined using different measurement methods. In addition, the exposure of erythrocytes to these stresses was different when assessed by different methods (Leverett et al., 1972; Rooney, 1970; Szwast et al., 2012; Williams et al., 1970).

It is also important that there is a large variation in the erythrocyte stamina depending on the donor (Nevaril et al., 1968), which means that all considerations carried out on responses of biological material refer only to the average values of the selected property.

Many scientific publications describe blood damage as a consequence of physical stress over years, i.e. (Bludszuweit, 1995; Gregoriades et al., 2000; Jones, 1995; Leverett et al., 1972; Nevaril et al., 1968; Pohorecki et al., 2001; Sutera and Mehrjardi, 1975). In these papers, one can find considerations that are not directly related to the artificial heart.

However, one can find papers in the literature concerning haemolysis inside the artificial heart. Song et al. (2003) assessed haemolysis by tracking a set of particles and their stress history. They used Computational Fluid Dynamics (CFD) for this purpose. Also Arvand et al. (2001) and Apel at al. (2001) have used CFD for haemolysis in their evaluation of artificial hearts.

Computational Fluid Dynamics (CFD) is a method used in medical device technology in particular when modelling the operation of artificial hearts (Bluestein, 2017). Pulsatile blood pumps have been modelled by e.g., Kim et al. (1992) and Okamoto et al. (2003). Nonpulsatile rotary blood pumps have been modelled by e.g. Medvitz et al. (2011), Mitamura et al. (2011) and Wang et al. (2008). CFD calculations refer to blood velocity distribution, mechanical stress distribution in different sites inside the artificial heart and heat exchange with external environment. The aforementioned papers of Arvand et al. (2001) and Song et al. (2003) also concern haemolysis assessment inside the artificial heart. However, their approach differs from the approach presented in this paper.

\section{NEW METHOD FOR HAEMOLYSIS ASSESSMENT}

Inside a rotary blood pump one can expect that the stress that acts on erythrocytes is induced in several ways. Such stress occurs as a result of collisions between erythrocytes and impeller blades and between two erythrocytes, and as well as an effect of cell-flow interactions (Pohorecki et al., 2001). 
The best way to evaluate haemolysis could be a direct calculation of hydrodynamic stresses inside the artificial heart (Apel et al., 2001; Bluestein and Mockros, 1969; Gregoriades et al., 2000). This is impossible by exclusively using CFD. But it is possible by an indirect approach because a relationship between energy dissipation $(\varepsilon)$ and hydrodynamic stresses exist. Bluestein and Mockros (1969) showed that there is a linear relationship in a log-log plot between haemolysis and energy dissipation rate. One can deduce it by analyzing the equations (1) and (2), which concern viscous and inertial subrange of turbulent spectrum, respectively (Bałdyga and Bourne, 1995):

$$
\begin{gathered}
\tau=\rho \cdot \sqrt{\varepsilon \cdot v} \text { for } d<\lambda_{k} \\
p=C \cdot \rho \cdot(\varepsilon \cdot d)^{\frac{2}{3}} \text { for } d>\lambda_{k}
\end{gathered}
$$

where

$$
\lambda_{k}=\frac{v^{0.75}}{\varepsilon^{0.25}}
$$

Having in mind relations given by Eqs. (1) - (3), our method of haemolysis assessment is reduced to calculations of energy dissipation which occurs in the artificial heart.

To obtain energy dissipation values, the Large Eddy Simulations (LES) method has been used. Tavoularis et al. (2003) and Yoganathan et al. (2005) recognized LES as a very promising tool in modeling phenomena inside the artificial heart.

\section{LARGE EDDY SIMULATIONS (LES)}

A new promising approach to solve equations related to turbulent flow is known as "Large Eddy Simulations techniques (LES)". In LES, a set of equations is derived from the Navier - Stokes equations by performing a spatial averaging. It is different when compared to time averaging in RANS (Reynolds-averaged Navier-Stokes equations) technique. The averaged equations contain stress terms that must be evaluated through modeling to achieve closure. The equations solved in LES are developed by "filtering" Navier - Stokes equations. This process removes small spatial scales. The resulting equations describe the evolution of large eddies and contain the subgrid-scale stress tensor that represents the effects of the unresolved small scales. Leonard (1974) proposed to decompose, flow variables into large (filtered, resolved) and sub-grid (residual) scales, as follows:

$$
v_{i}=\bar{v}_{l}+v_{i}
$$

The filtered variable is defined in the general case by the convolution integral:

$$
\bar{v}_{l}\left(x_{1}, x_{2}, x_{3}\right)=\iiint\left[\prod_{\mathrm{j}=1}^{3} G_{\mathrm{j}}\left(x_{\mathrm{j}}, x_{\mathrm{j}}^{\prime}\right)\right] v_{\mathrm{i}}\left(x_{1}^{\prime}, x_{2}^{\prime}, x_{3}^{\prime}\right) d x_{1}^{\prime} d x_{2}^{\prime} d x_{3}^{\prime}
$$

over the entire flow domain, where $x_{i}$ and $x_{i}$ are position vectors and $\mathrm{G}$ is the general filter function. The function $\mathrm{G}$ is normalized by requiring that:

$$
\iiint\left[\prod_{j=1}^{3} G_{j}\left(x_{j}, x_{j}^{\prime}\right)\right] d x_{1}^{\prime} d x_{2}^{\prime} d x_{3}^{\prime}=1
$$

Several filter functions G, have already been examined (Aldama, 1990), but the volume-averaged "box" filter is most frequently used with finite-difference and finite-volume methods. The box filter function is given by:

$$
G_{j}\left(x_{j}-x_{j}^{\prime}\right)=\left\{\begin{array}{cc}
\frac{1}{\Delta_{j}} & \left|x_{j}-x_{j}\right| \leq \frac{\Delta_{j}}{2} \\
0 & \text { otherwise }
\end{array}\right.
$$

using this filtering function we obtain 


$$
\bar{v}_{l}(\mathbf{x}, t)=\frac{1}{\Delta^{3}} \int_{x_{1}-\frac{1}{2} \Delta x_{1}}^{x_{1}+\frac{1}{2} \Delta x_{1}} \int_{x_{2}-\frac{1}{2} \Delta x_{2}}^{x_{2}+\frac{1}{2} \Delta x_{2}} \int_{x_{3}-\frac{1}{2} \Delta x_{3}}^{x_{3}+\frac{1}{2} \Delta x_{3}} v\left(x_{1}-x_{1}^{\prime}, x_{2}-x_{2}^{\prime}, x_{3}-x_{3}^{\prime}\right) d x_{1}^{\prime} d x_{2}^{\prime} d x_{3}^{\prime}
$$

where

$$
\Delta=\sqrt[3]{\Delta_{1} \Delta_{2} \Delta_{3}}
$$

and $\Delta_{1}, \Delta_{2}, \Delta_{3}$ are increments in $x_{1}, x_{2}, x_{3}$, respectively. Filtering the Navier - Stokes and the continuity equation gives:

$$
\begin{gathered}
\frac{\partial \overline{v_{j}}}{\partial x_{j}}=0 \\
\frac{\partial \bar{v}_{l}}{\partial t}+\frac{\partial \overline{v_{l} v_{J}}}{\partial x_{j}}=-\frac{\partial \bar{p}}{\partial x_{i}}+\frac{\mu}{\rho} \frac{\partial^{2} \bar{v}_{l}}{\partial x_{k}^{2}}
\end{gathered}
$$

The system cannot be solved for both $\bar{v}_{l}$ and $\overline{v_{l} v_{\jmath}}$, so that the convective flux should be represented by decomposed variables, as follows:

$$
\overline{v_{\imath} v_{J}}=\bar{v}_{\imath} \bar{v}_{J}+\tau_{i j}
$$

Introducing Eq. (12) into (11), the following equation can be obtained:

$$
\frac{\partial \overline{v_{l}}}{\partial t}+\frac{\partial \bar{v}_{l} \overline{v_{j}}}{\partial x_{j}}=-\frac{\partial \bar{p}}{\partial x_{i}}+\frac{\mu}{\rho} \frac{\partial^{2} \overline{v_{l}}}{\partial x_{k}^{2}}-\frac{\partial \tau_{i j}}{\partial x_{j}}
$$

where $\tau_{i j}$ is the sub-grid-scale (SGS) stress tensor:

$$
\tau_{i j}=\left(\overline{\bar{v}_{l} \overline{v_{J}}}-\overline{v_{l}} \overline{v_{J}}\right)+\left(\overline{v_{l} \overline{v_{J}}}+\overline{\overline{v_{l}} v_{J}}\right)+\left(\overline{v_{l} v_{J}}\right)
$$

The first term in parentheses on the right-hand side of the Eq. (14) is known as "Leonard stress", the second term as "cross-term stress", and the third term as "Reynolds stress". Note, that if time averaging has been employed instead of filtering, the first two terms would be zero leaving only the Reynolds stress. To get the solution of Eq. (13), the effect of the sub-grid-scale (SGS) stresses must be modeled. The earliest and simplest model was proposed by Smagorinski (1963). It takes the form of mixinglength or gradient diffusion model with the length proportional to the filter width. Thus, the SGS stress tensor is represented by:

$$
\tau_{i j}=2 \mu_{T} S_{i j}
$$

where $S_{i j}$ is the rate of strain tensor and

$$
\mu_{T}=\rho\left(C_{s} \Delta\right)^{2} \sqrt{2 S_{i j} S_{i j}}
$$

where $C_{s}$ is a well-known Smagorinsky constant which has to be prescribed as a fixed value in the entire integration domain with value from 0.1 to 0.24 , or can be determined as a function of time and space by a dynamic procedure originally proposed by Germano et al. (1991) and later improved by several authors, e.g. by Lilly (1992). For models with a constant value of $C_{s}$, Van Driest - type exponential damping function results in a form such as (Moin and Kim, 1982):

$$
l=C_{s} \Delta \sqrt{\left[1-e^{\left(\frac{-y^{+}}{A^{+}}\right)^{3}}\right]}
$$

It is required in order to take into account the reduction of the sub-grid length $l$ near solid walls. $y^{+}$from Eq. (17) is defined by

$$
y^{+}=\frac{y \rho}{\mu} \sqrt{\frac{\tau_{s}}{\rho}}
$$


With $A^{+}=25$. Recently, in many published investigations, the fixed parameter version of the Smagorinsky model has been applied. A theoretical value for the $C_{s}=0.165$ can be derived for homogeneous isotropic flows (Lilly, 1967). For practically anisotropic shear flows, smaller values of $C_{s}$ were found to be more appropriate. The well-established standard constant $C_{s}=0.1$ is implemented in standard version of the LES model into CFD package FLUENT. The solution of the filtered Navier - Stokes equations gives the time-dependent solution for the resolved variables. In applications that operate nominally at a steady state, we are usually interested in the time-mean motion of flow in a steady sense. The time averaging of filtered equations is needed to obtain the time-averaged values of the variables.

In the literature, there is no paper where LES is used for haemolysis assessment in the artificial heart. However, this method has already been used for modeling of non-Newtonian blood flows through blood vessels (Molla and Paul, 2009).

\section{RESULTS AND DISCUSSION}

Using FLUENT package 6.13 and LES method, computational results for different centrifugal pump impeller geometries have been obtained. In view of Eqs. (1) and (2), we have focused on the analysis of energy dissipation inside the modeled artificial heart. On the basis of computational results, we concluded that haemolysis will be greater where areas of higher values of energy dissipation are noticed or where the average value of energy dissipation inside the artificial heart is higher than that of other geometries. In calculation, blood rheological properties have been approximated by Newtonian fluid with a density of $1 \mathrm{~g} / \mathrm{cm}^{3}$ and a viscosity of $3.26 \mathrm{mPas}$.

Figures 1-3 present computational results of energy dissipation obtained for 3 different impeller geometries and for 2 different rotation speeds.

We have analysed an impeller with curved blades (Fig.1) and impellers with curved channels (Fig. 2 and Fig. 3). A common analysis of the results obtained for all impellers allows one to note the following observations. The average value of energy dissipation in the artificial heart for all impeller geometries is always less for the lower rotation speed. This statement is rather obvious.

For the impeller with curved blades (Fig.1), the highest values of energy dissipation are observed at the end of blades. Thus, according to the authors' proposed method, haemolysis will take place in particular in these areas. To reduce destruction of blood elements, the geometry at the end of the blades should be optimised and corrected.

For impellers with curved channels, the highest values of energy dissipation are noted inside the channels and around the periphery of the impeller, in particular at the blood outflow from the channels. This is obviously related to the high speed of blood flowing through a narrow channel. To reduce the destruction of blood elements, the geometry of the end of the channels should be corrected.

From comparing the results shown in Fig. 2 and Fig.3, it can be concluded that the average value of energy dissipation in the pumps with such impellers is lower for the impeller whose channels are bent in the opposite direction to the rotation of the impeller (Fig. 3). It is clearly seen when comparing Fig. $2 \mathrm{~b}$ and Fig. $3 \mathrm{~b}$. The difference in the values of energy dissipation is 10 times.

The exemplary calculation results, which are given in this paper, indicate that the lowest haemolysis should be expected for the impeller with curved channels that are bent in the opposite direction to the rotation of the impeller. The results of these calculations confirm the results of the experimental studies of one of the authors conducted on prototype pumps using human blood (Szwast, 2009). 
a)

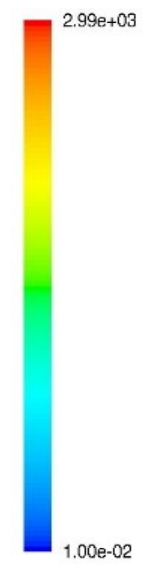

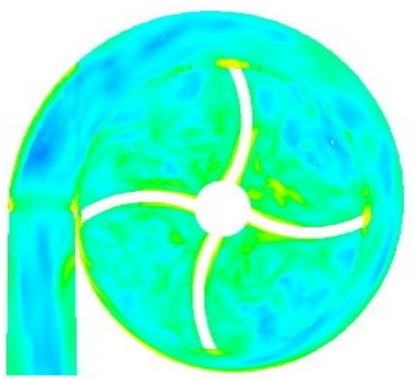

b)
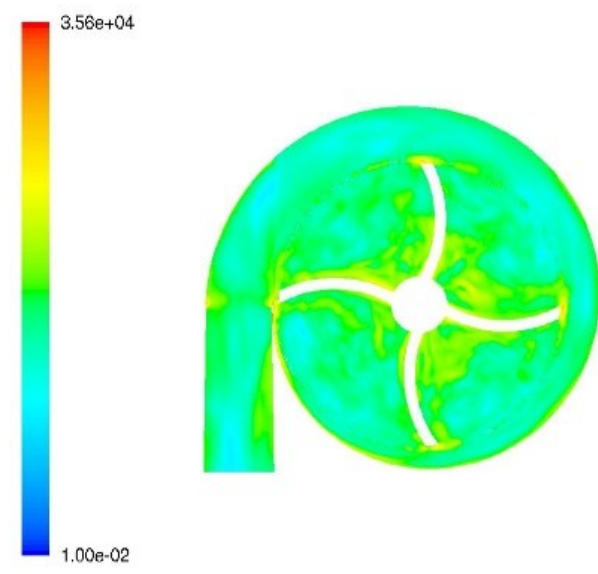

Fig. 1. Energy dissipation $\left[\mathrm{m}^{2} / \mathrm{s}^{3}\right]$ in artificial heart, geometry 1: a) $1000 \mathrm{rpm}$, b) $3000 \mathrm{rpm}$. Open impeller with left-curved blades

a)

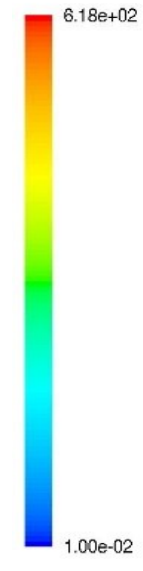

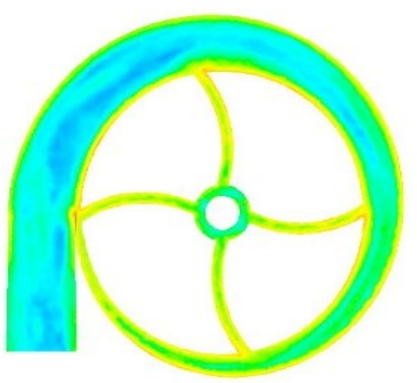

b)
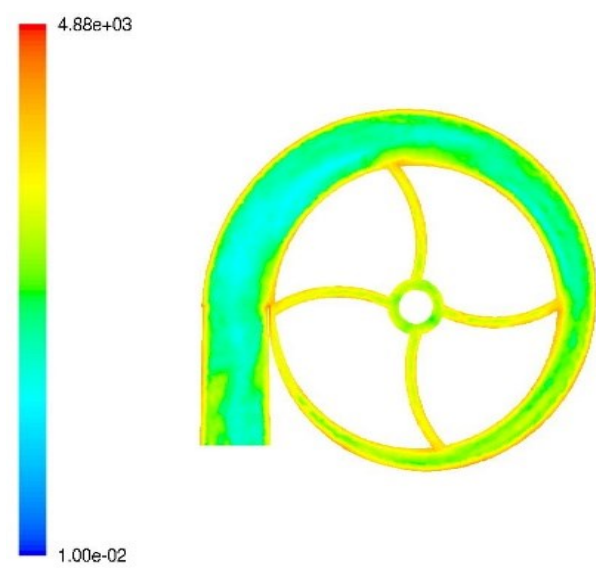

Fig. 2. Energy dissipation $\left[\mathrm{m}^{2} / \mathrm{s}^{3}\right]$ in artificial heart, geometry 2: a) $1000 \mathrm{rpm}$, b) $3000 \mathrm{rpm}$. Closed impeller with left-curved channels

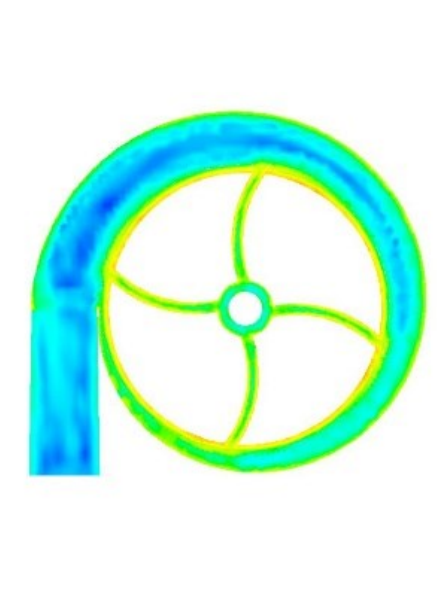

b)

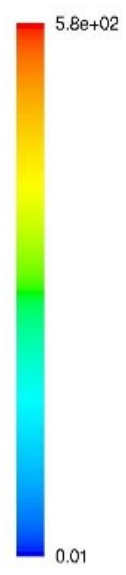

a)

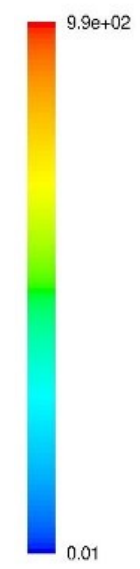

Fig. 3. Energy dissipation $\left[\mathrm{m}^{2} / \mathrm{s}^{3}\right]$ in artificial heart, geometry 3: a) $1000 \mathrm{rpm}$, b) $3000 \mathrm{rpm}$. Closed impeller with right-curved channels 


\section{CONCLUSIONS}

This paper presents a new method for assessing blood cell haemolysis based on stress analysis that has been caused by the impeller of a centrifugal pump in an artificial heart. CFD modeling using the LES model allows one to obtain reliable results of the value of energy dissipation inside the artificial heart. These results may be directly associated with the destruction of blood elements inside the pump. The haemolysis assessment tool proposed in this paper can be used for optimisation of new geometries of impellers for pumps, which will be used as heart assist devices.

\section{SYMBOLS}

$\begin{array}{ll}A^{+} & \text {damping constant } \\ C & \text { constant } \\ C_{S} & \text { Smagorinski constant } \\ d & \text { particle diameter, } \mathrm{m} \\ G_{j} & \text { box filter function } \\ p & \text { stress in inertial subrange, } \mathrm{Pa} \\ \bar{p} & \text { filtered value of pressure, } \mathrm{Pa} \\ S_{i j} & \text { strain tensor } \\ v_{i} & \text { sub-grid scale velocity, } \mathrm{m} / \mathrm{s} \\ \bar{v}_{j} & \text { jth component of velocity, } \mathrm{m} / \mathrm{s} \\ \bar{v}_{l} & \text { filtered value of velocity, } \mathrm{m} / \mathrm{s} \\ x_{j} & \text { position vector } \\ x_{j} & \text { position vector } \\ y^{+} & \text {dimensionless parameter defined by Eq. }(18) \\ \Delta & \text { increment in space, } \mathrm{m} \\ \Delta_{j} & \text { increment in } x_{j}, \mathrm{~m} \\ \varepsilon & \text { turbulent energy dissipation rate per mass unit, } \mathrm{m}^{2} \cdot \mathrm{s}^{-3} \\ \lambda_{k} & \text { Kolmogorov scale, } \mathrm{m} \\ \mu & \text { dynamic viscosity, Pa } \cdot \mathrm{s} \\ \mu_{T} & \text { turbulent viscosity, Pa } \cdot \mathrm{s} \\ \nu & \text { kinematic viscosity, } \mathrm{m}^{2} \cdot \mathrm{s}^{-1} \\ \rho & \text { density, } \mathrm{kg} \cdot \mathrm{m}^{-3} \\ \tau & \text { stress in viscous subrange, Pa } \\ \tau_{i j} & \text { viscous stress tensor } \\ \tau_{S} & \text { viscous stress on wall } \\ & \end{array}$

\section{REFERENCES}

Aldama A.A., 1990. Filtering techniques for turbulent flow simulation. Lecture Notes in Engineering. Vol. 56, Springer-Verlag, Berlin.

Apel J., Paul R., Klaus S., Siess T., Reul H., 2001. Assessment of hemolysis related quantities in a microaxial blood pump by computational fluid dynamics. Artif. Organs, 25, 341-347. DOI: 10.1046/j.1525-1594.2001.025 005341.x.

Arvand A., Hormes M., Reul H., 2005. A validated computational fluid dynamics model to estimate hemolysis in a rotary blood pump. Artif. Organs, 29, 531-540. DOI: 10.1111/j.1525-1594.2005.29089.x.

Bałdyga J., Bourne J.R., 1995. Interpretation of turbulent mixing using fractals and multifractals. Chem Eng Sci., 50, 381-400. DOI:10.1016/0009-2509(94)00217-F. 
Bludszuweit C., 1995. Model for a general mechanical blood damage prediction. Artif. Organs, 19, 583-589. DOI: $10.1111 / \mathrm{j} .1525-1594.1995 . t b 02385 . x$.

Bluestein D., 2017. Utilizing Computational Fluid Dynamics in cardiovascular engineering and medicine-What you need to know. Its translation to the clinic/bedside. Artif. organs, 41, 117-121. DOI: 10.1111/aor.12914.

Bluestein M., Mockros, L.F., 1969. Hemolytic effects of energy dissipation in flowing blood. Med. Biol. Eng. Comput., 7, 1-16. DOI: 10.1007/BF02474665.

Garatti A., Bruschi G., Colombo T., Russo C., Lanfranconi M., Milazzo F., Vitali E., 2008. Clinical outcome and bridge to transplant rate of left ventricular assist device recipient patients: comparison between continuous-flow and pulsatile-flow devices. Eur. J. Cardiothorac. Surg., 34, 275-280. DOI: 10.1016/j.ejcts.2008.02.019.

Germano M., Piomelli U., Moin P., Cabot W.H., 1991. A dynamic subgrid-scale eddy viscosity model. Phys. Fluids A: Fluid Dyn., 3, 1760-1765. DOI: 10.1063/1.857955.

Gregoriades N., Clay J., Ma N., Koelling K., Chalmers J.J., 2000. Cell damage of microcarrier cultures as a function of local energy dissipation created by a rapid extensional flow. Biotech. Bioeng., 69, 171-182. DOI: 10.1002/(SICI)1097-0290(20000720)69:2.

Jones S.A., 1995. A relationship between Reynolds stresses and viscous dissipation: Implications to red cell damage. Ann Biomed Eng, 23, 21-28. DOI: 10.1007/BF02368297.

Kim S.H., Chandran K.B., Chen C.J., 1992. Numerical simulation of steady flow in a two-dimensional total artificial heart model. J. Biomech. Eng., 114, 497-503. DOI: 10.1115/1.2894101.

Leonard A., 1975. Energy cascade in large-eddy simulations of turbulent fluid flows. Adv. Geophys., 18, $237-248$. DOI: $10.1016 / \mathrm{S} 0065-2687(08) 60464-1$.

Leprince P., Bonnet N., Rama A., Leger P., Bors V., Levasseur J.P., Gandjbakhch I., 2003. Bridge to transplantation with the Jarvik-7 (CardioWest) total artificial heart: A single-center 15-year experience. J. Heart Lung Transplant., 22, 1296-1303. DOI: 10.1016/S1053-2498(03)00036-6.

Leverett L.B., Hellums J.D., Alfrey C.P., Lynch E.C., 1972. Red blood cell damage by shear stress. Biophys $J .$, 12, 257. DOI: 10.1016/S0006-3495(72)86085-5.

Lilly D.K., 1967. The representation of small-scale turbulence in numerical simulation experiments, In: Goldstine H.H. (Ed.), Proceedings of the IBM Scientific Computing Symposium on Environmental Sciences. IBM Forum No. $320-1951,195-210$.

Lilly D.K., 1992. A proposed modification of the Germano subgrid-scale closure method. Phys. Fluids A: Fluid Dyn., 4, 633-635. DOI: 10.1063/1.858280.

Medvitz R.B., Boger D.A., Izraelev V., Rosenberg G., Paterson E.G., 2011. Computational fluid dynamics design and analysis of a passively suspended Tesla pump left ventricular assist device. Artif. Organs, 35, 522-533. DOI: 10.1111/j.1525-1594.2010.01087.x.

Mitamura Y., Takahashi S., Amari S., Okamoto E., Murabayashi S., Nishimura I., 2011. A magnetic fluid seal for rotary blood pumps: effects of seal structure on long-term performance in liquid. J Artif. Organs, 14(1), 23-30. DOI: $10.1007 / \mathrm{s} 10047-010-0526-8$.

Moin P., Kim J., 1982. Numerical investigation of turbulent channel flow. J. Fluid Mech., 118, $341-377$. DOI: $10.1017 / \mathrm{S} 0022112082001116$.

Molla M.M., Paul M.C., 2009. LES of non-newtonian physiological blood flow. 1st International Conference on Mathematical and Computational Biomedical Engineering - CMBE2009. June 29 - July 1, 2009, Swansea, UK.

Nevaril C.G., Lynch E.G., Alfrey C.P., Hellums J.D., 1968. Erythrocyte damage and destruction induced by shearing stress. J. Lab. Clin. Med., 71(5), 784-790.

Okamoto E., Hashimoto T., Inoue T., Mitamura Y., 2003. Blood compatible design of a pulsatile blood pump using computational fluid dynamics and computer-aided design and manufacturing technology. Artif. Organs, 27, 61-67. DOI: 10.1046/j.1525-1594.2003.07183.x.

Pohorecki R., Bałdyga J., Ryszczuk A., Motyl T., 2001. Erythrocyte destruction during turbulent mixing. Biochem. Eng. J., 9, 147-154. DOI: 10.1016/S1369-703X(01)00135-8.

Rooney J.A., 1970. Hemolysis near an ultrasonically pulsating gas bubble. Science, 169(3948), 869-871. DOI: 10.1126/science.169.3948.869.

Saito S., Nishinaka T., 2005. Chronic nonpulsatile blood flow is compatible with normal end-organ function: Implications for LVAD development. J Artif. Organs, 8, 143-148. DOI: 10.1007/s10047-005-0295-y.

Smagorinsky J., 1963. General circulation experiments with the primitive equations: I. The basic experiment. Mon. Weather Rev., 91, 99-164. DOI: 10.1175/1520-0493(1963)091. 
Song X., Throckmorton A.L., Wood H.G., Antaki J.F., Olsen D.B., 2003. Computational fluid dynamics prediction of blood damage in a centrifugal pump. Artif. Organs, 27, 938-941. DOI: 10.1046/j.15251594.2003.00026.x.

Sutera S.P., Mehrjardi M.H., 1975. Deformation and fragmentation of human red blood cells in turbulent shear flow. Biophys. J., 15, 1-10. DOI: 10.1016/S0006-3495(75)85787-0.

Szwast M., 2009. Selected experimental and computational aspects of artificial heart acting as a centrifugal pump. PhD Thesis.

Szwast M., Suchecka T., Piątkiewicz W., 2012. Mathematical model for biological cell deformation in a cylindrical pore. Chem. Process Eng., 33, 385-396. DOI: 10.2478/v10176-012-0034-x.

Tavoularis S., Sahrapour A., Ahmed N.U., Madrane A., Vaillancourt R., 2003. Towards optimal control of blood flow in artificial hearts. Cardiovascular Eng., 8, 20-31. DOI: 10.1046/j.1525-1594.2003.00026.x.

Wang Y., Song X., Ying C., 2008. Applications of traditional pump design theory to artificial heart and CFD simulation. Front. Energy Power Eng. China, 2, 504-507. DOI: 10.1007/s11708-008-0059-5.

Williams A.R., Hughes D.E., Nyborg W.L., 1970. Hemolysis near a transversely oscillating wire. Science, 169(3948), 871-873. DOI: 10.1126/science.169.3948.871.

Yoganathan A.P., Chandran K.B., Sotiropoulos F., 2005. Flow in prosthetic heart valves: state-of-the-art and future directions. Ann. Biomed. Eng., 33, 1689-1694. DOI: 10.1007/s10439-005-8759-z.

Received 29 November 2016

Received in revised form 15 May 2017

Accepted 16 May 2017 